\title{
Fuzzy comprehensive assessment method is based on the transmission lines of the analytic hierarchy process sewage flash
}

\author{
Wang Qiang, Song Shuting, Zhang Huiming \\ The Department of information technology \\ Shanxi Electric Power Corporation \\ Taiyuan,China \\ 15333666585@189.cn
}

\begin{abstract}
Transmission line flashing will cause large area and long time of blackouts, it happens in the working voltage, and it poses serious threat to the electric power system. With the development of industry,the increase of power grid capacity and rated voltage level,there are more and more flashing accidents occurred in the power system of power transmission and transformation equipment external insulation,also the damage that they bring about to our economy is getting larger.Aiming at a series of problems caused by transmission line flashing,this paper comes up with an assessment method of transmission line flashing,it improves the forecast accuracy and efficiency so as to ensure the safety operation of transmission.
\end{abstract}

Keywords: analytic hierarchy process; transmission line; pollution flashover; condition assessment

\section{INTRODUCTION}

Power contaminant flashover is the flashover and trip phenomenon, which happens when weather conditions appropriate, as the result of insulator dirty accumulation from the air pollutant[1]. Contaminant flashover accident accounts for only $30 \%$ of the power grid accidents, occurrence frequency after lightning, but that the economic losses it caused is eight to ten times as lightning disasters. Therefore, quick and accurate forecast and early warning of grid flashover disasters, become important content in disaster prevention and reduction of the grid work.

Insulator contaminant flashover is a complicated process, from clean insulator polluting and damping to leak and partial discharge, then to flashover, which includes physical, chemical, and other comprehensive effect. Contaminant flashover generally because When the insulator has accumulated dirty and damped, the flashover occurs because of the thermal effect the dielectric breakdown of the less conductive things.

Filth of insulator surface comes from atmospheric environment and pollution sources. There are so many types of pollution sources, salt spray, dust, metal powder of industrial zone, ash, etc. The impurity air suspended particles contaminate the insulator through subsiding on the insulator surface, electric field polarization attraction, fog and dew adsorption, and then the change of air temperature, sunlight, heating baking of the surface leakage current. Due to various natural conditions of accidental factors, and the influence of the insulator skirt edge shape, the distribution of the electric

\author{
Yao Dahai \\ Smart Grid Dep \\ Huada Tianyuan (Beijing) Electric Porwer Tecnaology \\ Co., LTD \\ Beijing, China \\ acprimer.yao@gmail.com
}

field, the installation position, the degree of contamination of the surface is different. Generally, salt density of lower surface is larger than upper surface.

All sorts of unclean mostly include conducting material (soluble or not soluble) and moisture absorption material. When the insulator surface in fog, dew, drizzle and industrial water mist, salt fog, the unclear layer adsorbs water and dissolves conductive material, becoming conductive solution. When the unclean layer moist enough, it will form a conductive layer throughout the insulator surface. Due to the low resistance of the conductive layer, there is leakage current in normal working voltage. Because of the uneven contaminant absorption, thus the attached conductive solution is different, leading to the uneven of surface resistance and leakage current. The large leakage current drys filthy and causes local overheating, thus it will increase resistance of the conductive layer and concentrate the electric field, causing partial discharge and producing pulse leakage current. Electric field intensity in the arc decreases with increasing of arc and discharge changes the distribution of the electric field, which increase the field intensity of the undried unclean area. Thus the leakage current increases, and partial overheating appear again, producing partial discharge. Along with the repetition and development of partial discharge, wet and dirty area's total resistance, which leads to the series-arc, reduces. Thus it increases the leakage current. When the field intensity reaches the limited value, arc will across through the insulator surface, leading to contamination flashover. When the power system is overvoltage, flashover process will be simplified, and time will be shorten.

The process of contamination flashover is not the electric breakdown of air gap, instead it is the thermal breakdown process requiring the accumulation of energy and times. and the process is random.

\section{INFLUENCE FACTOR OF TRANSMISSION LINE POLLUTION FLASHOVER}

It has been found that, many factors can contribute to the contamination flashover of transmission lines. In addition to the general public meteorological factors, there also are insulator's internal factors.

As shown in Figure 1: 


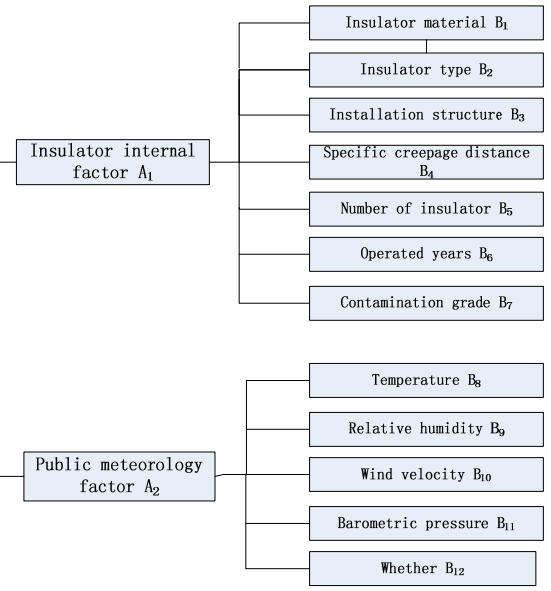

Figure 1. Contamination evaluation system

\section{THE EstABlishment OF EVAluAtion MODEL}

\section{A. Method and steps of fuzzy comprehensive evaluation}

1) Method of fuzzy comprehensive evaluation

Fuzzy comprehensive evaluation quantifies the fuzzy indicator relating to what need be judged, through structuring scaled fuzzy subset (namely, determine membership). Then, use the fuzzy transformation principle to synthesize the indicators [9].

\section{2) Step of Establishment}

a) Determining the factors domain of evaluation object Assuming there are $\mathrm{p}$ evaluation indicators

$$
u=\left\{u_{1}, u_{2}, \cdots \cdots, u_{p}\right\}
$$

b) Determine the evaluation grade theory field

$$
v=\left\{v_{1}, v_{2}, \cdots \cdots, v_{p}\right\}
$$

Namely the degrees set. Each grade corresponds to a fuzzy subset.

c) Establish fuzzy relation matrix $R$

After structuring the scaled fuzzy subset, we need to quantify each factor

$$
u_{i}(i=1,2, \cdots \cdots, p)
$$

That is, determine the membership $\left(R \mid u_{i}\right)$ of scaled fuzzy subset from the aspect of each single factor, and then get fuzzy relation matrix: $R=\left[\begin{array}{c}R \mid u_{1} \\ R \mid u_{2} \\ \cdots \\ R \mid u_{p}\end{array}\right]=\left[\begin{array}{cccc}r_{11} & r_{12} & \cdots & r_{1 m} \\ r_{21} & r_{22} & \cdots & r_{2 m} \\ \cdots & \cdots & \cdots & \cdots \\ r_{p 1} & r_{p 2} & \cdots & r_{p m}\end{array}\right]_{p . m}$

In the matrix $\mathrm{R}$, the $i_{t h}$ line and $j_{\text {th }}$ column element $r_{i j}$ indicates the membership of factor $u_{i}$ affiliate to grade $v_{j}$ of fuzzy subset. said some things to see "from the factors of level of membership. To describe the performance of a

certain factor $u_{i}$, we use the fuzzy vector $\left(R \mid u_{i}\right)=\left(\begin{array}{llll}r_{i 1} & r_{i 2} & \cdots & r_{i m}\end{array}\right)$. While other evaluation methods may use the actual value of a indicator to describe. Thus, from this aspect, fuzzy comprehensive evaluation requires more information. [10] factor

d) Determining the weight vector of the evaluation

In the fuzzy comprehensive evaluation, the weight vector of evaluation factors:

$$
A=\left(a_{1}, a_{2}, \cdots \cdots, a_{p}\right)
$$

The element $a_{i}$ of the weight vector $A$ is essentially the membership of factor $u_{i}$ regrading to fuzzy subset. This paper used the analytic hierarchy process(AHP) to determine the relative importance order among the evaluation indicators. Then, determine the weight coefficient, and then normalize before synthesizing. That is,

$$
\sum_{i=0}^{p} a_{i}=1, a_{i}>0, i=0,1,2 \cdots, n
$$

e) Synthesize result vector of the fuzzy comprehensive evaluation

Use appropriate operator to synthesize $A$ and $R$, then get comprehensive evaluation result vector $B$.Namely:

$$
A \circ R=\left(a_{1}, a_{2}, \cdots \cdots, a_{p}\right)\left[\begin{array}{cccc}
r_{11} & r_{12} & \cdots & r_{1 m} \\
r_{21} & r_{2} & \cdots & r_{2 m} \\
\cdots & \cdots & \cdots & \cdots \\
r_{p 1} & r_{p 2} & \cdots & r_{p m}
\end{array}\right]=\left(b_{1}, b_{2}, \cdots \cdots, b_{m}\right)=B
$$

In the expression, $b_{j}$ is the result of the operation of $A$ with the $j_{\text {th }}$ column of $R$. It indicates the membership of the object of study affiliate to $v_{j}$ degree fuzzy subset on the whole.

f) Analysis the result of the fuzzy comprehensive evaluation vector

The most commonly used method is the maximum membership degree principle. But in some cases, it will be some very reluctantly for a lot of information lost, and even result into unreasonable evaluation results. We put forward weighted average method for membership grade method, sorting studied multi-objects based on position of its degree.

\section{B. Determine weight with AHP}

\section{1) $\mathrm{AHP}$}

Getting weight is key to comprehensive evaluation. Analytic hierarchy process is an effective method to determine the weight coefficient. Especially suitable for those complex problem which are hard to analysize with indicators [11]. It divided each factor in the complicated problem into interrelated orderly layer. According to the 
fuzzy judgment of objective reality, quantitatively present the of the relative importance of each layer. And then determine the weight coefficient which relate to the all elements' relative importance order with mathematical methods.

\section{2) Steps of AHP}

a) Determine a goal and evaluation factors

Assuming there are $\mathrm{p}$ evaluation indicators

$$
u=\left\{u_{1}, u_{2}, \cdots \cdots, u_{p}\right\}
$$

b) Structure evaluation matrix

Value of evaluation matrix element reflects the people's recognition of the relative importance of each elements. Generally adopting scale method of 1-9 division and its reciprocal . But when importance of compared element can be indicated by practically meaningful ratio, it should be put into the matrix. Thus, we can get the judgement matrix

$$
S=\left(u_{i j}\right)_{p \times p}
$$

\section{c) Calculate judgment matrix}

Using MATLAB software to calculate the largest eigenvalue $\lambda_{\max }$, and the corresponding eigenvector $A$ of the judgment matrix. $A$ is the sort of the importance of each evaluation factor, and is the distribution of the weight coefficient as well.

d) Consistency test

To have consistency test of judgment matrix for inspection, it is necessary to calculate the consistency indicator $C I=\frac{\lambda_{\max }-n}{n-1}$, and the average random consistency indicator $R I$. That is to use random method to structure 500 sample matrix. The construction method is randomly fill the upper triangular of sample matrix of the with the divisions and their reciprocal, in which the value of main diagonal position always keeps as 1 and the number of the corresponding transposed position is the reciprocal of their corresponding position. Then calculate the consistency indicator for each random sample matrix, and the average value of $C I$ is the average random consistency indicator $R I$ [12]. When

$$
C R=\frac{C I}{R I}<0.10
$$

the random consistency ratio, we consider that result of ordering with AHP have satisfactory consistency. In other words, the distribution of the weight coefficient is reasonable. Otherwise, we have to adjust the elements value of judgment matrix to redistribution coefficient.

\section{MODEL SOLUTION}

\section{A. Multi-scale fuzzy comprehensive evaluation indicator of transmission line contamination flashover}

Fuzzy evaluation method is very practical when there are importance differences among evaluation indicators. There are two multi-scale fuzzy comprehensive evaluation methods: namely one step method (one-time comprehensive evaluation) and multistep method ( fuzzy evaluation step by step). In this paper we adopt multistep method.

The figure 1 shows that insulator state evaluation indicator system consists of two first layer indicators and 12 second layer indicators. The indicators are measured by Likert scale method. Use semantics scale to have four measurement scales: very good, good, middle, bad. To facilitate the calculation, we will subjectively quantify the semantics evaluation scale and in turn assignment for $4,3,2$, 1. The designed of the quantitative standard of evaluations is shown in table 1.

TABLE I. QUANTITATIVE EVALUATION OF GRADING STANDARDS

\begin{tabular}{lcc}
\hline Value of evaluation & Comment & Scale \\
\hline$x_{i}>3.5$ & very good & $E 1$ \\
$2.5<x_{i} \leq 3.5$ & good & $E 2$ \\
$1.5<x_{i} \leq 2.5$ & middle & $E 3$ \\
$x_{i} \leq 1.5$ & bad & $E 4$ \\
\hline
\end{tabular}

\section{B. AHP Steps for indicator weight}

1) Determining the evaluation object set

$\mathrm{P}=\{$ The prediction of insulator contamination flashover state $\}$

2) Construct the judgment factor set

$$
u=\left\{u_{1}, u_{2}\right\}=
$$

\{insulator internal factor, pubulic meteorology factor $\}$

3) Determining the judgment degree domain

Determining the judgment degree domain, namely, establish evaluation set:

$\mathrm{v}=\left\{\mathrm{v}_{1}, \mathrm{v}_{2}, \mathrm{v}_{3}, \mathrm{v}_{4}\right\}=\{$ very good, good, middle, bad $\}$

4) Calculation of first layer indicator weight

Two primary weight of factor indicators. We adopt AHP method to get the indicator weight. Construct judgement $\underset{\text { matrix }}{ } S=\left(u_{i j}\right)_{p \times p}$, namely:

$$
S=\left[\begin{array}{ll}
1 & 5 \\
\frac{1}{5} & 1
\end{array}\right]
$$

Use Mathematica software [15] to calculate the largest eigenvalue of judgment matrix $S$, get $\lambda_{\max }=2$. Calculate the consistency indicator for consistency test:

$$
C I=\frac{\lambda_{\max }-n}{n-1}=\frac{2-2}{2-1}=0
$$

Average random consistency index $R I=1.24$ 。 Random consistency ratio:

$$
C R=\frac{C I}{R I}=\frac{0}{1.24}=0<0.10
$$

So the think consistency of analytic hierarchy sort is satisfactory. In other words, the distribution of the weight 
coefficient is very reasonable. Correspondingly,the eigenvector is:

$$
A_{0}=(0.9806,0.1961)
$$

So the think consistency of analytic hierarchy sort is satisfactory. In other words, the distribution of the weight coefficient is very reasonable. Correspondingly,the eigenvector is:

$$
A_{0}=(0.9806,0.1961)
$$

Normalize the vector, get

$$
A=(\mathbf{0 . 8 3 3 3 , 0 . 1 6 6 7 )}
$$

5) Calculation of second indicator weight

Similarly, we still use hierarchical analysis method to evaluate the indicator weight. Respectively structure their judgment matrix for each secondary indicator. Then use Mathematica software to calculation the eigenvalue and consistency test, getting reasonable weight coefficient.

The judgement matrix of seven internal factor indicators

of the insulator: $\left[\begin{array}{ccccccc}1 & \frac{1}{3} & \frac{1}{3} & 7 & 1 & 5 & 9 \\ 3 & 1 & 1 & 7 & 1 & 5 & 9 \\ 3 & 1 & 1 & 7 & 1 & 5 & 9 \\ \frac{1}{7} & \frac{1}{7} & \frac{1}{7} & 1 & \frac{1}{7} & \frac{1}{3} & 7 \\ 1 & 1 & 1 & 7 & 1 & \frac{1}{3} & 9 \\ \frac{1}{5} & \frac{1}{5} & \frac{1}{5} & 3 & 3 & 1 & 9 \\ \frac{1}{9} & \frac{1}{9} & \frac{1}{9} & \frac{1}{7} & \frac{1}{9} & \frac{1}{9} & 1\end{array}\right]$

Based on the above methods for weight, its normalized vector is:

\section{$(0.0479,0.0294,0.0332,0.2110,0.0642,0.0939,0.5204)$}

The judgement matrix of five public meteorology factor indicator of insulator: $\left\{\begin{array}{ccccc}1 & 3 & 1 & 3 & \frac{1}{3} \\ \frac{1}{3} & 1 & \frac{1}{3} & \frac{1}{3} & \frac{1}{5} \\ 1 & 3 & 1 & 3 & 3 \\ \frac{1}{3} & 3 & \frac{1}{3} & 1 & \frac{1}{3} \\ 3 & 5 & \frac{1}{3} & 3 & 1\end{array}\right\}$

Its normalized vector is:

$(0.2032,0.0603,0.3434,0.1011,0.2919)$

\section{Multi-level fuzzy comprehensive evaluation of} transmission line contamination flashover

1) Weighted average of the fuzzy synthetic comprehensive evaluation of the transmission line contamination flashover

By using the weighted average $M(\bullet, \oplus)$ fuzzy synthetic operator, we synthesize $A$ and $R$ to get the fuzzy comprehensive evaluation result vector $B$. Fuzzy comprehensive evaluation commonly adopts choosing big or small algorithm. When there are more factors, the weight distributed to each factors is often very small. In the fuzzy synthetic operation, a lot information lost, leading to indistinguishable and unreasonable results(namely model failure) [16]. Therefore, to solve the mentioned problem, here we use the weighted average fuzzy synthesis operator. Calculation formula is:

$$
\left.b_{i}=\sum_{i=1}^{p} a_{i} \cdot r_{i j}\right)=\min \left(1, \sum_{i=1}^{p} a_{i} \cdot r_{i j}\right), j=1,2, \cdots ; m
$$

In the formular, $a_{i}, b_{i}, r_{i j}$ respectively are the membership of $j$ grade , the weight of $i_{t h}$ the evaluating indicator and the membership of indicator $i$ affiliate to $j$ grade.

2) The result factor of multi-level fuzzy comprehensive evaluation

Put a group of contamination flashover simulation statistics data into the model, and calculate the fuzzy comprehensive evaluation vector for each layer.

a) Judgement vector of insulator internal factor

$$
A_{1}=a \circ R
$$

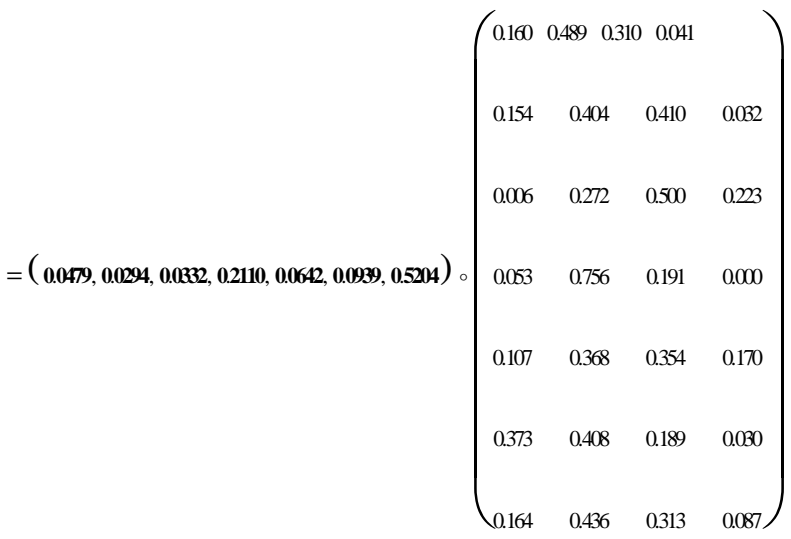

$=(0.150309,0.458948,0.311525,0.079172)$

Normalized vector:

$(0.084,0.226,0.499,0.197)$

b) Judgement vector of pubic meteorology factor

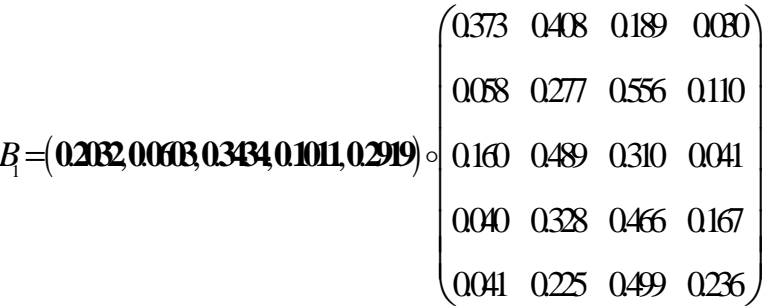

$$
\begin{aligned}
& =(0.082535,0.225865,0.498879,0.197111)
\end{aligned}
$$

Normalized vector:

$$
(0.084,0.226,0.499,0.197)
$$

c) Comprehensive judgement vector

$$
A=(\mathbf{0 . 8 3 3 3 , 0 . 1 6 6 7}) \circ\left(\begin{array}{llll}
0.020 & 0.231 & 0.501 & 0.188 \\
0.048 & 0.226 & 0.277 & 0.236
\end{array}\right)
$$

d) Ranking the comprehensive evaluation value $V_{A}=4 \times 0.150+3 \times 0.459+2 \times 0.312+1 \times 0.079=2.68$ 


\section{$V_{B}=4 \times 0.084+3 \times 0.226+2 \times 0.499+1 \times 0.197=2.09$}

From the above results, according to the valuation grading standards shown in table1, that the of table 1, "internal factors" evaluation indicator of insulator is ranked as "good", belonging to grade E2, and the "public meteorology factors" evaluation indicator of insulator is ranked as "middle", belonging to the grade E3.The value of comprehensive evaluation for the whole is

\section{$V=4 \times 00336+3 \times 03034+2 \times 06112+1 \times 00518=23188$}

It indicates that overall evaluation of transmission line is "middle", belonging to degrade E3. Insulator state monitoring is required in the operation, so that we can take timely decontamination measures.

The most commonly used method is the maximum membership degree principle, but this method use is conditional, for its validate problem, which may result into unreasonable evaluation. Putting forward weighted average principle for membership grade method, analyze evaluation results of each layer's evaluation indicators. The results of this method differ from the result of the maximum membership degree principle methods a bit, but the results conform to the actual situation.

\section{CONCLUSION}

Applying fuzzy mathematics to comprehensively the possibility of transmission line contaminant flashover, often appear the problem that results is not easy to be distinguished, because of more evaluation indicators and the choosing big and small algorithm. Based on the weighted average evaluation, we achieve good results.

When analyze the comprehensive evaluation results, the commonly used maximum membership degree principle method has validity problem. To analyze the results and making comparison sort for multi-indicators, we adopt the weighted average principle,and the result is satisfactory.

Presently, the weights are mostly given by experts based on experience, which includes serious human interference relatively, leading to the uncertain of evaluation results. This paper the use analytic hierarchy process (AHP) to determine weigh for fuzzy comprehensive evaluation. This method is logical, practical and systematic and can accurately get weight coefficient.

The fuzzy comprehensive evaluation of transmission line contamination flashover prediction based on analytic hierarchy process (AHP) is achieve a good result. The established model conforms to the actual situation, solution for the model is simple. It has a good application prospect and promotional value.

\section{REFERENCE}

[1] Hu, Yonghong, Enhui He, Method of Comprehensive evaluation, Beijing:Science, 2000: 167-188.

[2] Li Zuoyong, Environmental quality assessment principle and method, Beijing:Chemical industry, 2004:69-133.

[3] Yao Shuyou, Research of insulator contamination statement evaluation and prediction.

[4] Yang Lunbiao, Fuzzy mathematics application and principle, Guangzhou: South China university of science and technology, 2000,67-80.

[5] Xie,Jijian, Chengping Liu, Fuzzy mathematic method and application, Wuhan: South China university of science and technology, 2000:205-211.

[6] Ge, jun, Lunying Ge, "Analytic hierarchy process to determine the weight indicator of water quality". Contemporary Architecture, 2003, 3(1), 22-23.

[7] Guizhou province meteorological research institute, Wei Li, Forecast trial for Guizhou power contamination flashover risk level, 1999.

[8] Li , Angui, Zhihong Zhang, Yan Meng, Fuzzy mathematics with application, Wuhan: Metallurgical industry,2003:144-146.

[9] Wang, Xinzhou, Fuzzy space information processing, Wuhan: Wuhan University,2003:130-131.

[10] Li, Shiyong, Engineering fuzzy mathematic with application, Harbin: Harbin Institute of Technology, 2004:101-108.

[11] Tong, Chunsheng, The theory and method introduction of system engineering, Beijing: National Defence Industry,2000: 185-186.

[12] Jiang, qiyuan, Mathematical Model, Beijing: Higher Education, 2005:225-231.

[13] Ning, Xiaoqiu, Fuzzy Mathematic Principle and Method, Xuzhou: China Mining University, 2004:203-208.

[14] Liu, Yuangao, Yaoru Liu, Mathematica 4.0 Practical guide, Beijing: National Defence Industry, 2000:65-68.

[15] Changzhi Meteorological Agency, ZhengWang Wang, Power Flashing Accident analysis of meteorological conditions, 2005.10.

[16] Guizhou Meteorological Institute, Qishun Liu, Meteorological Causes of Power Equipment Contamination Flashover disaster, 1997.

[17] Shanxi Electrical Power Scientifical Academy, Guohua Zhou, Influence Factor Analysis of Shanxin Electrical Power System External Insulation Filth Classification, 2004.12.

[18] Fujing Electrical Power Company, Han lin, Insulator Contamination Flashover Prediction Method Based on Artificial Neural Network.

[19] Zhang ,lin, Ruxin liu, "Transmission Line Insulator Contamination Flashover Mechanism ".Journal of Electric Power, 1999, 14(2):87-90

[20] Hebei Electrical Power Academy, Question and Answer for Grid Contamination Flashover Prevention Technology. 